\title{
Handwriting Based Interface for Communication
}

\author{
Santanu Chaudhury, Aditya Khandelia \\ II-405, Multimedia Lab, IIT Delhi, Hauz Khas, New Delhi, India -110016 \\ schaudhury@gmail.com, adityakhandelia@gmail.com
}

\begin{abstract}
Handwriting has been conventionally used for input by applying handwriting recognition. In this paper we propose to use handwriting without recognition as a temporal medium of communication in synchronization with other media like audio and video. This ensures that the interfaces developed are language independent and provide rich, natural and intuitive interaction. We present multiple applications exploiting this concept.
\end{abstract}

Keywords: Handwriting, human computer interface, interactive devices, pen computing.

\section{INTRODUCTION}

Handwriting is one of the most basic tools for communication as it forms an integral part of the learning process. On one hand, it is a tool for communication, and on the other hand, an enchanting art. Apart from the written message, handwriting reflects personality of an individual and thus makes the message more personalized. It is independent of the language. Sentences in any language, formulas, diagrams can be all written with a pen. By writing with a pen, one can express one's thinking easily. One does not have to change the device (pen) to draw text, formulas, figures, tables and so thinking is not interrupted by the actions for writing. This nature of writing is suited for creative work and communication. Also the basic device, pen, is a scalable interface unlike mouse or keyboard, i.e., it can be used commonly between PDAs, desktop tablets and large interactive whiteboards.

Thus we see that handwriting has the potential to provide an intuitive and easy to grasp method for interfacing with the device. The concept of pen computers is a testimony to the same. While it has been recognised for long that writing combined with gestures can free us from the tyranny of a keyboard, mediocre quality of handwriting recognition has been a major obstacle. Despite lot of efforts, users report the new systems to be slow, inaccurate and demanding of user attention.

In this paper we propose to use writing as temporal medium of communication without recognition. This idea provides multiple advantages. It removes the constraints imposed by recognition framework like language and vocabulary dependence, syntax, semantics and constraints on writer like lifting pen between strokes, entering text in a specific box etc. Also such a framework provides user with a blank-page like space to write on with no constraints of writing in standard left-to-right, top-to-bottom method. Thus it provides a highly intuitive language independent interface. Also by incorporating temporal information, the user will get a powerful handwriting interface allowing him to draw diagrams, doodles figures etc all presented in temporally consistent fashion as desired by user and if required, synchronized with other audio-visual information. Combining this framework with multi-touch gesture based command system would provide users with a potent tool facilitating them in capturing their expressions in a much richer and natural way and ensuring that these will be reproduced in an equally immersive way to the recipient.

The rest of the paper is organized as follows. Section 2 talks about the literature survey of some of the recent work, section 3 contains some concept systems proposed/developed by us and finally we end with concluding remarks.

\section{LITERATURE SURVEY}


In recent times lot of work has been focused on pen based computing and handwriting oriented interfaces keeping in mind the advantages obtained over traditional systems. These applications focus on using different features of handwriting based on the intended purpose.

An overview of pen computing is given by Subrahmonia and Zimmerman [Subrahmonia, 2000]. They discuss the advantages of pen computing and provide an overview covering three aspects namely, pen input hardware, handwriting recognition and pen computer applications. They describe various pen tracking technologies like magnetic, electric, ultrasonic and optical used for taking input. Further, a framework for handwriting recognition is discussed taking into consideration noise removal, segmentation, feature extraction, recognition and linguistic processing.

Sano et.al.[Sano, 2008] propose a human computer interface for hand writing character. Their work focuses on kanji characters for which brush is typically used to write on paper. In this paper a new human-computer interface for hand writing character that uses ordinary papers and brush pen is developed. Strokes written by the brush pen are input into a computer by using a CCD camera and can be reconstructed and displayed as a character on the computer display at the realistic rate.

Another use of digital ink is proposed by Jian Wang[Wang, 2005]. They developed a software system and a hardware pen prototype that allows users to write and annotate on the MS Office printed documents with a digital pen. Inking, annotating, reading smart tags and searching are just some of the functionalities that they propose to provide. All handwriting information on the printed documents are captured digitally just like on tablet PC and synchronized with the original digital documents on PCs.

Some of the products based on similar principles are commercially available in the market. XLibris [5] is a prototype interface for reading at FX Palo Alto Lab. It uses a tablet display to show documents and provides user with paper functionalities like annotating, underlining etc.

In terms of whiteboard technologies, mimio [6] provides a lot of solutions. Mimio interactive allows user to control the computer by using a special pen-like pointing device as mouse on the display projected on the whiteboard. Mimio capture records the text written by user on the whiteboard (without time stamps) and allow saving it in various formats.

Our proposed idea is different from existing work in following ways (i) Use of handwriting without recognition as a data type itself rather than for input to other fields. (ii) Preserving of temporal characteristics of writing to provide for a more natural means of communication and (iii) exploring the possibility of synchronizing multiple data types using gestures for control. In the following section we conceptualize some potential applications which exploit these salient features.

\section{CONCEPT SYSTEMS}

\subsection{Asynchronous Communication}

Asynchronous communication refers to the mode of communication where the users involved need not be present simultaneously, for eg. email, voicemail etc. The traditional systems for this type of communication are basically built to handle only single type of data, voice for voicemail, text for email etc. Although email supports sending attachments, they cannot be synchronized with the text.

Here we present an integrated architecture which combines handwritten data (text in any language, doodles, figures etc) captured as temporal medium synchronized in time with other media such as audio and video, sending all in a single file format and not separately as attachments. The motivation for such architecture comes from the fact that while communicating, we usually speak with accompanying gestures, demonstrations and save time by drawing figures, doodles or writing key words etc. Thus a communication system that captures these simultaneously with temporal synchronization has the capability to provide users with an immersive, near-life communication experience. This can have applications in distance education wherein students do not have to be present at the same time for a lecture but can view it at their own convenient time and later respond with queries. Another application could be to view meetings, conferences or training sessions offline. In terms of personal communication, the idea has the capability to provide us with an entirely new email paradigm, allowing users a conversational style interaction experience. 
A prototype system called pátrá [Harit, 2007] has been developed by us. Pátrá is an integrated document architecture which incorporates handwritten illustrations captured and rendered in a temporal fashion synchronized with audio, video, text, and image data. The architecture of Pátrá

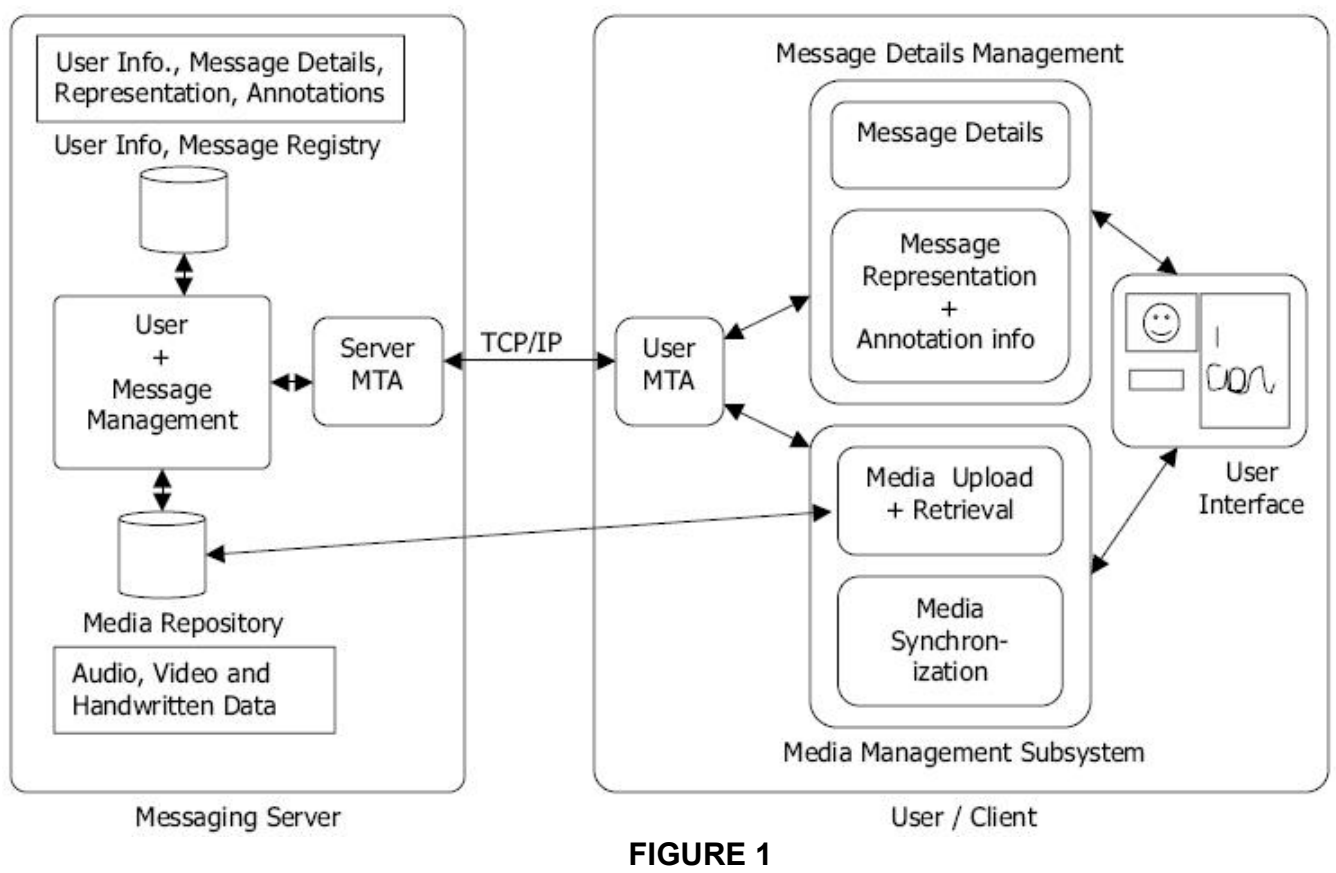

permits non-linear growth in the form of multiple hierarchically organized play streams. Semantic metadata is also an integral part of Pátrá which serves a useful purpose of organizing such documents in a collection. We have developed an email application in which the users are provided with an authoring and rendering environment to compose, view, and reply to messages in the form of Pátrá. The system architecture for Pátrá is shown in figure 1.

MPEG-4 has been chosen as the video data-encoding format in current implementation since it offers consistently high quality video at high and low bit rates and offers good error resilience. For audio coding, we have used the MPEG Layer-3 audio codec which is a highly evolved and mature audio encoding standard with an equally well supported open source effort. The current system design is flexible enough to allow change in the audio, video encoding formats. Change in the audio-video encoding formats does not affect the synchronization relations between the audio, video and the corresponding handwritten data. For handwriting information InkML i.e Ink Markup Language has been used which was developed by the Multimodal Interaction working group at W3C. Ink Markup Language supports a complete and accurate representation of digital ink and allows temporal representation through the use of timestamps.

\subsection{Meeting Board}

Meeting board is another application that provides a medium for communication which is based on recognizing handwriting as a data type instead of limiting it by applying handwriting recognition thereby opening up new and natural ways of expression. As in the case of previous application, here also we have exploited the idea of preservation of temporal characteristics of the handwritten data. This enhances the communication experience since preservation of temporal characteristics while rendering the data at user end give the feel that other person is writing infront of him in real time.

The basic idea behind the application is to provide the user with a large board area which is virtually shared at other remote locations. The user can write/draw the required message which is immediately synchronized with all the other locations. If required, the message can instead be stored with timestamps and then sent to be viewed later, i.e for asynchronous communication. Additionally, the receiver has the ability to annotate messages, highlight topics etc and insert these in specific temporal locations in a message.

Another salient feature provided by the application is integration of written and digital data. The user can have digital documents/media opened up alongside the written data and use gestures to carry out operations like dragging to different locations, selecting text etc. The gestures can be 
implemented by either using a touch screen or using a projector and a camera to convert any surface into a potential meeting board. The user can also make comments and annotations in the document using the pen just as he would if it were a printed document. The overall framework is shown in figure 2. The novelty of concept lies in the overall architecture which revolves around the use of handwriting as a temporal medium of communication.

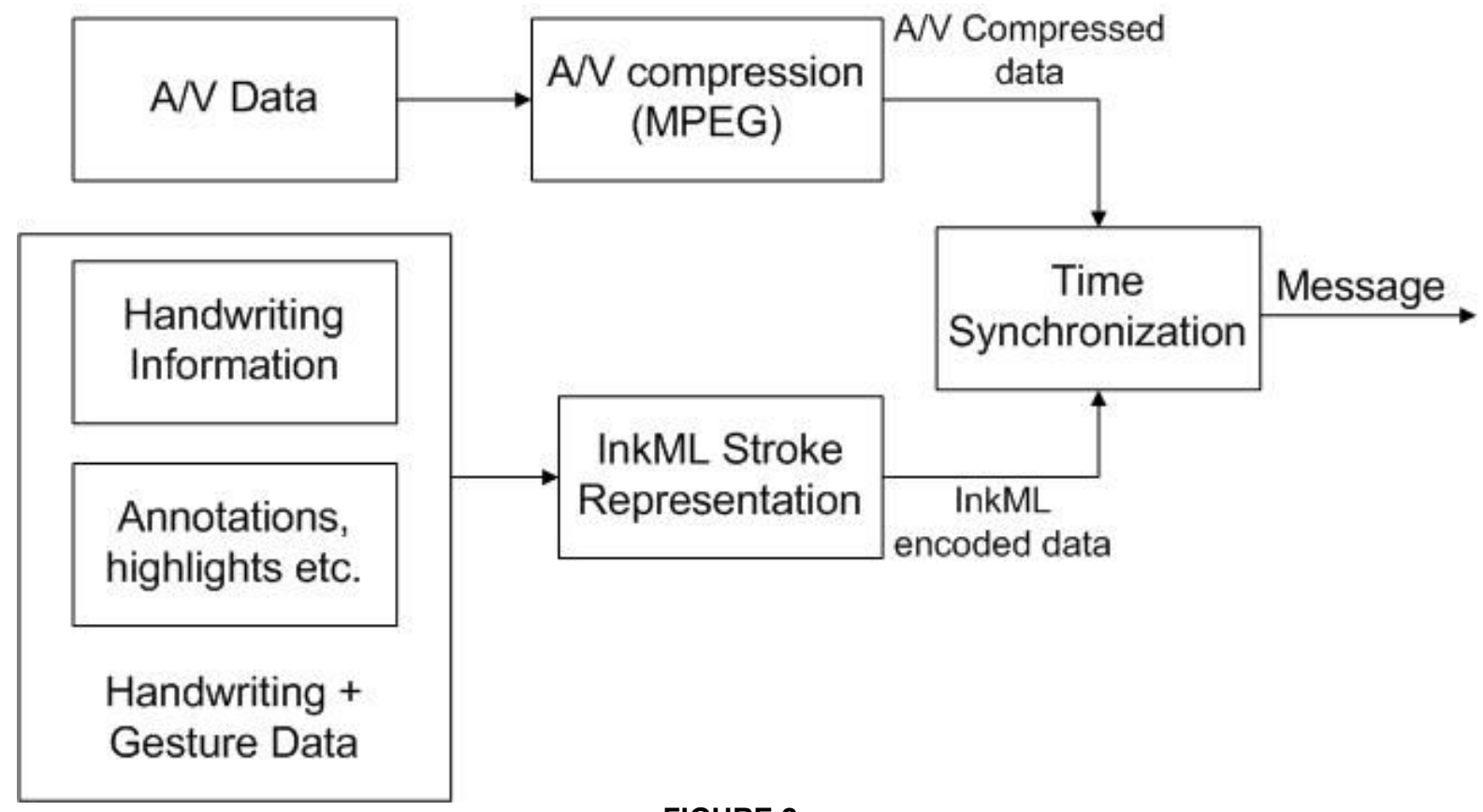

FIGURE 2

\subsubsection{Table-top display}

A parallel application to meeting board would be sharing of a large display are at the same location. Here the users can have their own workspace on a large display and there can be a common area for everyone to work on. The handwriting and gesture capabilities, like writing or document editing, would be same as that for meeting board with support for additional features like virtually "passing" a document to another user at the table by means of gestures. As in the case of meeting board, the display can be a plain surface with a projector and a camera for tracking gesture commands or a touch sensitive surface. The table top display is different from upcoming touch based computers in terms of the functionalities provided. Here the objective is to provide a large surface on which multiple users work simultaneously. The actual computing might take place at a remote location, with any available table working as display with the help of a projector.

\subsection{Combining Virtual and Real Documents}

Digital documents are often printed for reading, editing, sharing etc. However, a big disadvantage of doing this is that user's comments and annotations on the document are no longer synchronized with the original document and thus keeping track of the changes or sending them to another person becomes difficult.

To get around this difficulty we propose a framework to synchronize virtual and real documents. As the user makes changes on the document, a tracking pen provides all the co-ordinate information required to reconstruct the handwriting information. For this purpose any of the present tracking pen technologies can be used. A camera captures the original document image and the system uses document image retrieval to get the original document. The changes and annotations are then appended into it thus keeping the virtual and real document in synch. Thus the changes are available to the user as a virtual copy and can be easily shared with other remote users.

\subsection{SMS application}

The new generation of mobile phones coming in the market are increasingly choosing touch screen over the standard keyboard for input. The handwriting systems available on these devices depend on recognition, which as discussed, is not very user friendly. The application that we propose allows 
user to draw/write a message on a blank screen and send it via the sms protocol and not mms. This allows the user to freely use the available space to draw and write in whichever way he/she desires.

As per the requirement the data can be sent with timestamps thus ensuring that the information appears in time synchronized order. This allows for asynchronous communication, wherein the recipient can make comments and annotations in the message at required temporal locations and send it back, thus allowing for a much more natural method of communication.

The feasibility of such a system depends a lot on compression of handwriting data since a standard text message contains only 160 bytes. For this purpose, vector graphics based compression can be used. An additional advantage of using vector graphics compression would be that it provides scalability which is very important since mobile devices vary widely in terms of screen sizes and resolutions.

To test the idea we have developed a prototype in J2ME. The reasons for selecting J2ME are that most of the cell phones are Java enabled. J2ME has been designed for small, resource-constrained devices and it can be emulated on a PC. Applications successfully emulated on PC are easily upload able on phones. A slight drawback is that applications work rather sluggishly.

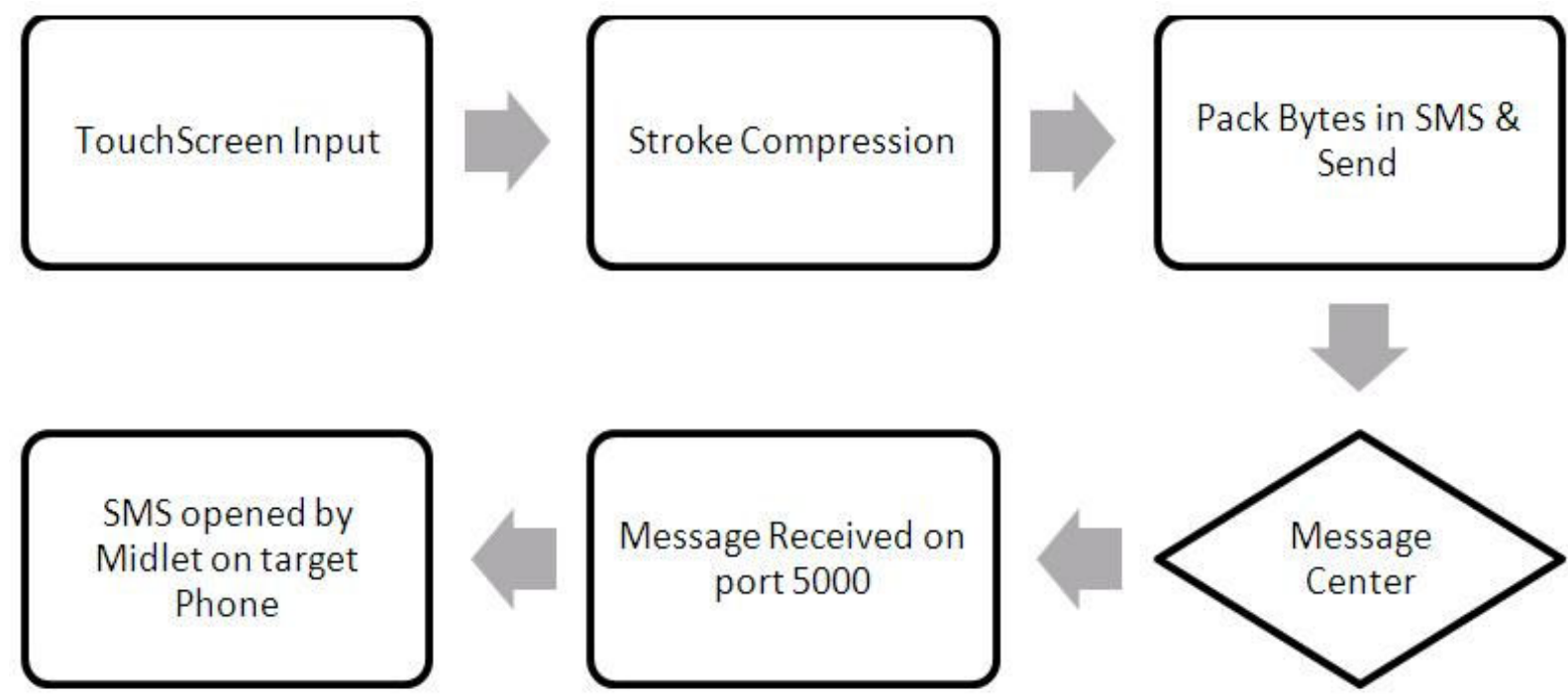

FIGURE 3

The overall framework has been shown in figure 3. First the user draws the required message on his Touch screen device. The message is then compressed, packed as bytes into message and sent. A vector graphics based compression algorithm has been used in which each stroke is compressed as follows:

- All sets of 3 successive points are checked for co linearity. The points are said to be colinear if the lines joining first and second point and second and third point do not differ in slope by more than +-5 degrees.

- If co linear, the middle point is eliminated.

- The first and last points are necessarily put into the output.

- The compressed array (consisting of start and end points for lines) is then packed as bytes and sent to the decoder.

The receiver identifies the message as associated with the decoder application, and launches it. The application decodes, and displays the message. An example is shown in figure 4. 


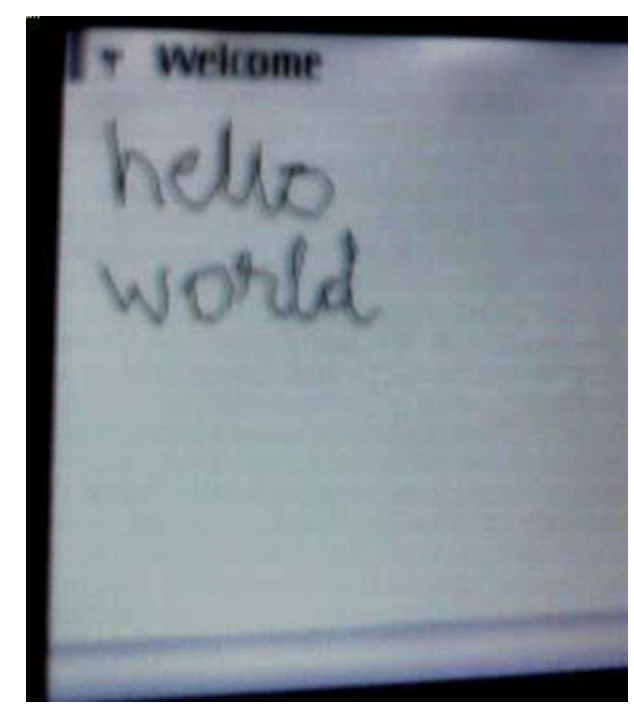

FIGURE 4

The novelty of this idea lies in the fact that it has the potential to change the way in which we approach text messaging. Traditionally text messaging, as the name suggests, is primarily built for handling text data. The data is input from a keyboard (virtual or real) and thus there is a constraint on language that can be used and the way it can be presented. However with this system, the user is free to scribble text, doodle, draw figures etc and send it via the cheap SMS protocol while preserving the arrangement (spatial and temporal) of components and the handwriting allowing for a more experiential and engaging communication.

\section{CONCLUSION}

The idea of using handwriting as temporal medium opens up new avenues for communication and interaction. It presents advantages over existing methods by providing intuitive, natural and life-like interfaces. Combining handwriting, audio and video in a time synchronized manner takes us very close to the way we interact in day to day life thus making our digital experience more human.

\section{REFERENCES}

[1] J. Subrahmonia and T. Zimmerman (2000) Pen computing: challenges and applications Proceedings of 15th International Conference on Pattern Recognition, 3-7 September, vol. 2, pp. 60-66.

[2] T. Sano, H. Ukida, and H. Yamamoto (2008) Design of human-computer interface for hand writing character IEEE International Instrumentation and Measurement Technology Conference, 1215 May, p. 594-597.

[3] J. Wang(2005) Pen computing: Digital ink and printed document Proceedings of the Eighth International Conference on Document Analysis and Recognition, 29 August - 1 September, vol. 1, p. 334.

[4] G. Harit, V. Mankar, and S. Chaudhury (2007) Patra: A novel document architecture for integrating handwriting with audio-visual information Ninth International Conference on Document Analysis and Recognition, 23-26 September, vol. 2, pp. 699-703.

[5] http://www.fxpal.com/?p=xlibris

[6] http://www.mimio.com/products/index.asp 